\title{
Clinically Relevant Hormone Treatments Fail to Induce Spinogenesis in Prefrontal Cortex of Aged Female Rhesus Monkeys
}

\author{
Daniel T. Ohm, ${ }^{1 \star}$ Erik B. Bloss, ${ }^{1 \star}$ William G. Janssen, ${ }^{1}$ Karen C. Dietz, ${ }^{1}$ Shannon Wadsworth, ${ }^{1}$ Wendy Lou, ${ }^{4}$ \\ Nancy A. Gee, ${ }^{5}$ Bill L. Lasley, ${ }^{5}$ Peter R. Rapp, ${ }^{6}$ and John H. Morrison ${ }^{1,2,3}$ \\ ${ }^{1}$ Fishberg Department of Neuroscience, The Friedman Brain Institute, Kastor Neurobiology of Aging Laboratory, ${ }^{2}$ Department of Geriatrics and Palliative \\ Care, and ${ }^{3}$ Graduate School of Biological Sciences, Mount Sinai School of Medicine, New York, New York 10029, ${ }^{4}$ Dalla Lana School of Public Health, \\ University of Toronto, Toronto, M5T 3M7 Ontario, Canada, ${ }^{5}$ Center for Health and the Environment and California National Primate Research Center, \\ University of California, Davis, California 95616, and 'Laboratory of Experimental Gerontology, National Institute on Aging, Baltimore, Maryland 21224
}

Preclinical animal models have provided strong evidence that estrogen (E) therapy (ET) enhances cognition and induces spinogenesis in neuronal circuits. However, clinical studies have been inconsistent, with some studies revealing adverse effects of ET, including an increased risk of dementia. In an effort to bridge this disconnect between the preclinical and clinical data, we have developed a nonhuman primate (NHP) model of ET combined with high-resolution dendritic spine analysis of dorsolateral prefrontal cortical (dlPFC) neurons. Previously, we reported cyclic ET in aged, ovariectomized NHPs increased spine density on dlPFC neurons. Here, we report that monkeys treated with cyclic E treatment paired with cyclic progesterone $(\mathrm{P})$, continuous E combined with $\mathrm{P}$ (either cyclic or continuous), or unopposed continuous E failed to increase spines on dIPFC neurons. Given that the most prevalent form of ET prescribed to women is a combined and continuous $\mathrm{E}$ and $\mathrm{P}$, these data bring into convergence the human neuropsychological findings and preclinical neurobiological evidence that standard hormone therapy in women is unlikely to yield the synaptic benefit presumed to underlie the cognitive enhancement reported in animal models.

\section{Introduction}

Working memory (WM) is dependent on neuronal networks located within the dorsolateral prefrontal cortex (dlPFC) (Goldman-Rakic, 1995). WM performance is known to be sensitive to aging, where impairments can be apparent in monkeys as early as middle-age (Moore et al., 2006; Wang et al., 2011), and can be linked to changes in endocrine hormone status (Roberts et al., 1997; Rapp et al., 2003; Lacreuse, 2006). Cognitive benefits associated with hormone therapy (HT) during perimenopause have been observed in women (Phillips and Sherwin, 1992; Kimura, 1995; Verghese et al., 2000); however, the precise relationship between HT and cognitive function remains poorly understood and controversial (Morrison et al., 2006).

Nonhuman primate (NHP) models have provided strong evidence that estradiol (E) therapy (ET) improves WM and en-

Received April 18, 2012; revised June 7, 2012; accepted June 25, 2012.

Author contributions: D.T.O., E.B.B., P.R.R., and J.H.M. designed research; D.T.O., E.B.B., W.G.J., K.C.D., S.W., N.A.G., B.L.L., and J.H.M. performed research; D.T.O., E.B.B., W.L., N.A.G., B.L.L., and P.R.R. analyzed data; D.T.O., E.B.B., P.R.R., and J.H.M. wrote the paper.

This work was supported by NIA Grant AG034794 (to E.B.B.); NIA Grants AG016765, AG006647, and AG010606 (to J.H.M.); and the NIA Intramural Research Program (to P.R.R.). We thank Drs. Mark Baxter, Patrick Hof, Dani Dumitriu, Yuko Hara, Jiandong Hao, and Rishi Puri for expert advice and assistance.

*D.T.O. and E.B.B. contributed equally to this work.

The authors declare no competing financial interests.

Correspondence should be addressed to Dr. John H. Morrison, Fishberg Department of Neuroscience, Box 1065, Mount Sinai School of Medicine, One Gustave L. Levy Place, New York, NY 10029. E-mail: john.morrison@mssm.edu. DOI:10.1523/JNEUROSCI.1881-12.2012

Copyright $\odot 2012$ the authors $\quad 0270-6474 / 12 / 3211700-06 \$ 15.00 / 0$ hances spinogenesis in dlPFC circuits (for review, see Voytko et al., 2009; Bailey et al., 2011). Results from the Women's Health Initiative Memory Study (WHIMS), however, concluded that combined and continuous estrogen and progestin HT actually "increased the risk for probable dementia in postmenopausal women aged 65 or older" (Shumaker et al., 2003). The discrepancy between preclinical results and the WHIMS findings could be based on several factors, including the characteristics of women studied in the WHIMS (who were older, had generally poorer health, and long postmenopause time periods without HT) as well as the specific formulation of HT (Gibbs and Gabor, 2003; Turgeon et al., 2004). From this perspective, animal models avoid numerous significant confounds and limitations found in clinical studies, and allow the direct study of the behavioral, cellular, and synaptic effects of HT. Furthermore, ovariectomizing (OVX) animals simulates natural menopause in women and ensures the most uniform baseline before hormone intervention.

We have previously reported that unopposed cyclic E treatment improved dlPFC-dependent cognitive performance (Rapp et al., 2003) and increased the density of the dendritic spines on dlPFC neurons in aged, OVX female monkeys (Hao et al., 2006, 2007). In contrast, the HT used in the WHIMS was continuous and combined formulations of multiple estrogenic compounds (i.e., Premarin) and a progestin (medroxyprogesterone acetate, i.e., Provera). To directly test whether the timing and compositions of HT improved dlPFC structural plasticity, we investigated cyclic and continuous regimens of $\mathrm{E}$ and progesterone $(\mathrm{P})$ in our 


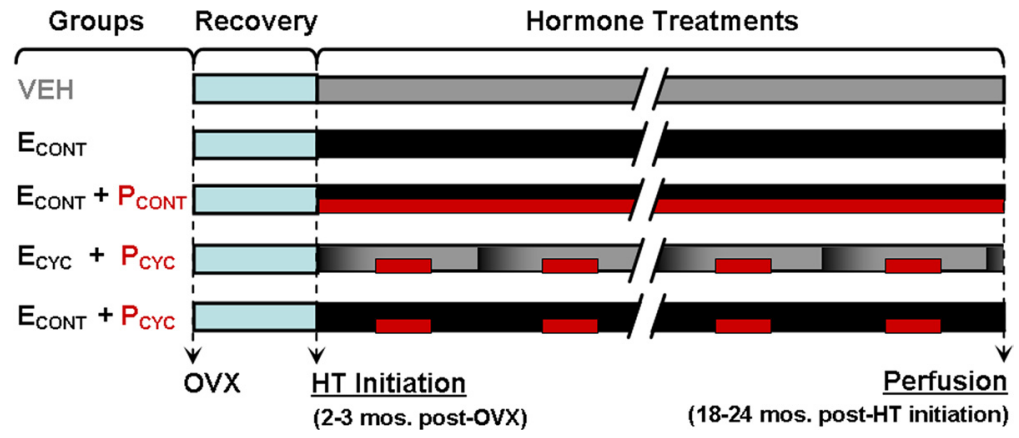

Figure 1. Experimental design of HT. Groups included: vehicle control, continuous E, continuous E plus continuous P, cyclic E every $28 \mathrm{~d}$ (black-to-gray gradient bars) plus cyclic $P$ for $10 \mathrm{~d}$ beginning $10 \mathrm{~d}$ after Einjection (red bars), and continuous E plus cyclic P $10 \mathrm{~d}$ of every 28 . See Results for treatment efficacy.

aging NHP model and analyzed morphological parameters of dIPFC pyramidal neurons. Our data show that, unlike the spinogenesis seen with unopposed cyclic E replacement, aged monkeys treated with cyclic $\mathrm{E}$ treatment paired with cyclic $\mathrm{P}$, continuous E combined with $\mathrm{P}$ (either cyclic or continuous), or unopposed continuous $\mathrm{E}$ failed to increase spines on dlPFC neurons. Together, these data provide compelling evidence that the standard forms of HT prescribed to women today may be ineffective in promoting or sustaining synaptic plasticity in aging neuronal circuits that mediate cognition.

\section{Materials and Methods}

Animals. Thirty-five OVX female rhesus monkeys (Macaca mulatta; age range, 17.7-25.7 years; mean age, $20.7 \pm 1.9$ years old at OVX) were used in this study. Animals were housed under conditions identical to previous studies (Rapp et al., 2003; Hao et al., 2006) and were given daily fruit treats in addition to a ration of monkey chow. All experiments were conducted in compliance with the National Institutes of Health Guidelines for the Care and Use of Experimental Animals approved by the Institutional Animal Care and Use Committee at the University of California, Davis.

OVX and hormone treatment. OVX surgery was performed as previously described (Rapp et al., 2003). After 2-3 months of post-OVX recovery, animals were assigned to blinded experimental groups consisting of the following conditions: vehicle $(\mathrm{VEH})$, continuous $\mathrm{E}\left(\mathrm{E}_{\mathrm{CONT}}\right)$, continuous $\mathrm{E}$ and continuous $\mathrm{P}\left(\mathrm{E}_{\mathrm{CONT}}+\mathrm{P}_{\mathrm{CONT}}\right)$, cyclic $\mathrm{E}$ and cyclic $\mathrm{P}$ $\left(\mathrm{E}_{\mathrm{CYC}}+\mathrm{P}_{\mathrm{CYC}}\right)$, and continuous $\mathrm{E}$ and cyclic $\mathrm{P}\left(\mathrm{E}_{\mathrm{CONT}}+\mathrm{P}_{\mathrm{CYC}}\right)$ (Fig. 1).

Hormone treatments were administered as follows: continuous $\mathrm{E}$ was delivered via SILASTIC capsule implants containing crystalline estradiol (Sigma) placed subcutaneously between the shoulder blades and changed every 3 months for animals in the $\mathrm{E}_{\mathrm{CONT}}, \mathrm{E}_{\mathrm{CONT}}+\mathrm{P}_{\mathrm{CONT}}$, and $\mathrm{E}_{\mathrm{CONT}}+\mathrm{P}_{\mathrm{CYC}}$ groups. Continuous $\mathrm{P}$ (micronized; Catalent Pharma Solutions) was given orally in $100 \mathrm{mg}$ capsules. Monkeys in the VEH and $\mathrm{E}_{\mathrm{CYC}}+\mathrm{P}_{\mathrm{CYC}}$ groups received implants of empty capsules, and intramuscular injections every $28 \mathrm{~d}$ consisting of either $1 \mathrm{ml}$ of sterile peanut oil or $100 \mu \mathrm{g}$ of estradiol cypionate (Wedgewood Pharmacy) in $1 \mathrm{ml}$ of sterile peanut oil. For monkeys in the $\mathrm{E}_{\mathrm{CYC}}+\mathrm{P}_{\mathrm{CYC}}$ and $\mathrm{E}_{\mathrm{CONT}}+\mathrm{P}_{\mathrm{CYC}}$ groups, the fruit treats contained micronized progesterone for 10 of every $28 \mathrm{~d}$, beginning $10 \mathrm{~d}$ after $\mathrm{E}$ or $\mathrm{VEH}$ injection; fruit given to $\mathrm{VEH}$ and $\mathrm{E}_{\mathrm{CONT}}$ monkeys never contained progesterone. These procedures ensured that all technical staff remained blind to treatments, which lasted $\sim 18-24$ months. Treatment efficacy was verified by chemiluminescent immunoassays of serum samples collected on the same set schedule (four samples collected quarterly) across all groups.

Perfusion and tissue collection. Animals were perfused as previously described (Hao et al., 2006). Perfusions were done $24 \mathrm{~h}$ after the last injection of $\mathrm{E}$ in the $\mathrm{E}_{\mathrm{CYC}}+\mathrm{P}_{\mathrm{CYC}}$ group, and on the corresponding day for all other treatments $\left(\sim 8-10 \mathrm{~d}\right.$ after cessation of $\mathrm{P}$ in $\mathrm{E}_{\mathrm{CONT}}+\mathrm{P}_{\mathrm{CYC}}$ groups). Animals were deeply anesthetized and perfused transcardially with ice-cold $1 \%$ paraformaldehyde in $0.1 \mathrm{M}$ PBS for $1 \mathrm{~min}$, followed by ice-cold 4\% paraformaldehyde in PBS for 12 min. After perfusion, the brain was immersionpostfixed for $6 \mathrm{~h}$ in $4 \%$ paraformaldehyde and $0.125 \%$ glutaraldehyde in PBS, and dissected into multiple standardized blocks, one of which contained the entire region surrounding the principal sulcus of the dlPFC (Brodmann's area 46). Frontal blocks containing area 46 were cut into $400-\mu \mathrm{m}$-thick sections in serial fashion on a Vibratome (VT1000S; Leica).

Intracellular injections and dendritic morphological analyses. Intracellular injections were performed as previously described (Hao et al., 2006). Pyramidal cells from layer III of area 46 were injected with 5\% Lucifer yellow (Invitrogen) under a direct current of 1-5 nA until the dye filled all distal branches of the dendritic tree (Fig. 2A). To be included for analysis, neurons had to satisfy three criteria: located inside layer III of Brodmann's area 46; complete filling of every branch of the dendritic tree based on observation of bright, well defined endings; and a sufficient distance from adjacent loaded cells to allow for unambiguous identification of dendritic branch origins and dendritic spines.

As in previous studies (Hao et al., 2006), pyramidal neurons were reconstructed using a Zeiss Axiophot 2 microscope equipped with a $40 \times$ objective (Plan-NEO, 1.3 NA), a motorized stage, video camera system, and Neurolucida morphometry software (MBF Bioscience) (Fig. 2B). For each monkey, five to eight pyramidal cells in area 46 were traced and exported to Neurolucida Explorer for morphological analysis.

Quantitative confocal analyses of spine density and spine morphology. High-magnification imaging was performed similar to previous reports (Hao et al., 2006), with minor modifications. Dendritic segments were systematically selected by placing concentric circles of 60 and $120 \mu \mathrm{m}$ in radius over the cell soma. Branches that intersected each circle were imaged using a Zeiss 510 confocal microscope equipped with an Argon laser, Zeiss $100 \times 1.4 \mathrm{NA}$ oil-immersion objective, and ZEN software. Confocal $z$-stacks were taken with voxel dimensions of $0.05 \times 0.05 \times 0.1$ $\mu \mathrm{m}$. Each animal had approximately five randomly selected neurons imaged, with an average of six apical segments and three basal segments per neuron captured for analysis. Dendritic segments used in the current study did not overlap with branches that could obscure spine origins; had no branch bifurcations within the $z$-stack; and were taken from secondary, tertiary, or quaternary branches.

Confocal stacks were deconvolved (Media Cybernetics, version 8.0.2) (Fig. 2C) and imported to NeuronStudio software for three-dimensional spine density, spine type, and spine size analyses (Dumitriu et al., 2011). Spines were classified as thin or mushroom if the ratio of their maximum head diameter to maximum neck diameter was $>1$.1. Spines that met this criterion and had a maximum head diameter $<0.47 \mu \mathrm{m}$ were classified as thin spines, while those with maximum spine head diameters $>0.47 \mu \mathrm{m}$ were classified as mushroom spines. Spines with a head:neck ratio $<1.1$ were classified as stubby spines. Filopodia, characterized by long, thin protrusions without a swelling at the distal end, were exceedingly rare and were scored as thin spines. To minimize bias, all analyses were done blind to the experimental condition.

Statistical analyses. For each morphometric parameter, mean values per subject were calculated and used to compute group averages for statistical comparison. Group differences in mean dendritic lengths and dendritic spine densities were analyzed using a one-way ANOVA followed by Bonferonni post hoc tests. Sholl data were analyzed using a two-way mixed-model repeated-measure ANOVA. Differences in the cumulative frequency of dendritic spine head diameters and spine volumes were analyzed with Kolmogorov-Smirnov tests. For all tests, $\alpha$ was set at 0.05 .

\section{Results}

\section{HT efficacy}

To validate the effectiveness of HTs, median values from serum $\mathrm{E}$ and $\mathrm{P}$ assays were calculated for each monkey across relevant 


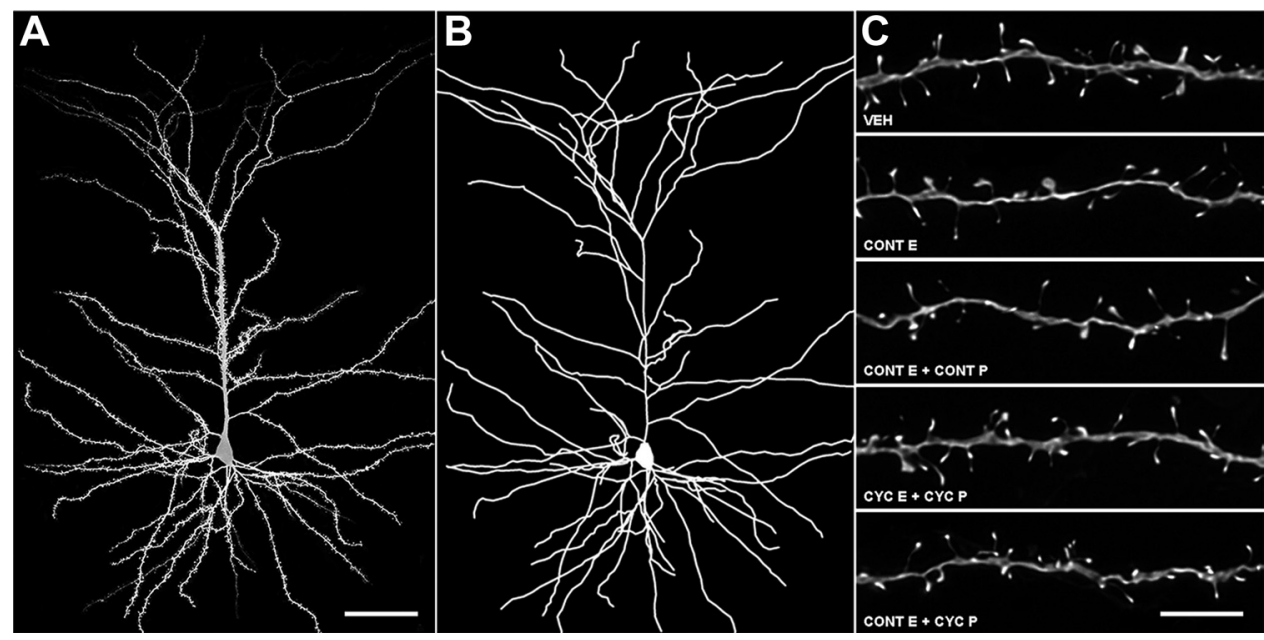

Figure 2. dIPFC neuronal reconstruction methodology. $\boldsymbol{A}$, Confocal image of a layer III monkey dIPFC neuron used in the present study. $\boldsymbol{B}$, Dendritic reconstruction of the same neuron. $\boldsymbol{C}$, Deconvolved confocal images of apical dendritic segments used in the present study. Scale bars: $A, 50 \mu \mathrm{m} ; \boldsymbol{C}, 5 \mu \mathrm{m}$.

sampling periods. Median data minimizes the influence of extreme values and adjusts for variations in duration of sampling across monkeys and for occasional missing values due to assay failure or lost samples ( $<1 \%$ of samples). Across the post-OVX study period, median serum E ranged between 7.09 and 35.95 $\mathrm{pg} / \mathrm{ml}$ in the $\mathrm{VEH}$ group and median serum $\mathrm{P}$ ranged from 0.12 to $0.59 \mathrm{ng} / \mathrm{ml}$, consistent with cessation of ovarian activity (Rapp et al., 2003). $\mathrm{E}_{\mathrm{CONT}}$ resulted in median serum E levels between 91.62 and $449.28 \mathrm{pg} / \mathrm{ml}$, although serum E levels were much higher immediately after implant change (233.92-1553.68 pg/ml $48 \mathrm{~h}$ after implant change). $\mathrm{P}_{\text {CONT }}$ replicated circulating levels of $\mathrm{P}$ approximate to the luteal phase, with median serum $\mathrm{P}$ values ranging from 2.79 to $3.40 \mathrm{ng} / \mathrm{ml}$. Median serum E levels from monkeys in the $\mathrm{E}_{\mathrm{CYC}}$ group ranged between 50.46 and 155.82 $\mathrm{pg} / \mathrm{ml} 48 \mathrm{~h}$ after E injection. These levels are lower than our previously published levels following injection (Rapp et al., 2003), which were sampled 9-24 h after injection rather than 48 h. Median serum E levels before the E injections ranged from 4.93 to $31.46 \mathrm{pg} / \mathrm{ml}$, consistent with cessation of ovarian function. Median serum levels of $\mathrm{P}$ from monkeys in the $\mathrm{P}_{\mathrm{CYC}}$ group ranged between 1.45 and $5.68 \mathrm{ng} / \mathrm{ml}$. Levels in this group before $P$ treatment ranged from 0.11 to $1.46 \mathrm{ng} / \mathrm{ml}$. Detailed analyses and plots of these data will be published elsewhere (M. Baxter, M. Roberts, N. Gee, B. Lasley, J. Morrison, and P. Rapp, unpublished observations).

\section{HT and dIPFC dendritic morphology}

We asked whether the HT altered the dendritic morphology of layer III dlPFC neurons. ANOVA found no effect of condition on mean total apical dendritic length $\left(F_{(4,30)}=0.1791, p>\right.$ 0.9 ; data not shown). Sholl analysis of apical dendrites revealed a main effect of distance from soma $\left(F_{(17,595)}=352.76\right.$, $p<0.05)$, no effect of condition $\left(F_{(4,30)}=0.18, p>0.9\right)$, and a significant interaction $\left(F_{(68,595)}=1.46, p<0.05\right)$. Bonferonni post hoc tests found no differences among the conditions at any specific distance in the apical dendritic tree. Likewise, analysis of basal dendrites revealed no effect of condition on mean total basal dendritic length $\left(F_{(4,30)}=0.5511, p>0.6\right.$; data not shown). Sholl analysis of basal dendrites revealed a main effect of distance from soma $\left(F_{(11,385)}=155.6, p<0.05\right)$, no effect of condition $\left(F_{(4,30)}=0.55, p>0.6\right)$, and no significant interaction $\left(F_{(44,385)}=0.71, p>0.9\right)$. Post hoc tests found no differences among the hormone conditions at any specific distance in the basal dendritic tree.

\section{HT and dIPFC dendritic spine density}

We next asked how the HT affected the density of dendritic spines. Previous studies have found that unopposed $\mathrm{E}_{\mathrm{CYC}}$ replacement in aged, female OVX monkeys results in an $\sim 30 \%$ increase in dendritic spines across both apical and basal dendritic branches (Hao et al., 2006). However, the analysis of overall spine density in the present study found no main effect of condition $\left(F_{(4,30)}=0.6758, p>0.6\right.$; Fig. $\left.3 A\right)$, and similar results were found when dendritic segments were separated into apical or basal domains (data not shown). Importantly, the aged VEH monkeys from our previous experiments (Hao et al., 2006, 2007) had spine densities indistinguishable from the current VEH-treated monkeys $(0.87 \pm 0.03$ vs $0.87 \pm 0.04$ spines $/ \mu \mathrm{m})$, confirming that our quantitative spine methodologies are highly reproducible across studies. Together, these data suggest that E's ability to increase spine density is dependent on both the schedule of the delivery and the presence of circulating $\mathrm{P}$.

Previous studies in aging monkeys have linked age-related cognitive decline with the loss of a particular class of spines on dlPFC neurons: the small, highly motile, thin spines (Dumitriu et al., 2010). Furthermore, our previous studies suggest unopposed cyclic E replacement in aged, OVX female monkeys improves cognitive performance and selectively increases the frequency of spines with smaller head diameters (Hao et al., 2006, 2007). Thus, we compared the density of spines as a function of spine type (i.e., thin, mushroom, or stubby spines; see Materials and Methods). ANOVA found no main effect of condition on thin spine density $\left(F_{(4,30)}=0.5281\right.$, $p>0.7$; Fig. $3 B)$, mushroom spine density $\left(F_{(4,30)}=0.2614, p>0.9\right.$; Fig. $3 C)$, or stubby spine density $\left(F_{(4,30)}=0.7193, p>0.5\right.$; Fig. $\left.3 D\right)$. These data strengthen the conclusion that despite effective hormone delivery, these regimens of HT were ineffective at inducing structural plasticity in aged NHP dlPFC dendritic spines.

\section{HT and dIPFC dendritic spine morphology}

Lastly, we asked whether the HT altered individual spine head diameters and spine head volumes. ANOVA found no effect of condition on mean head diameter $\left(F_{(4,30)}=0.2522, p>0.9\right)$, and analysis of spine diameter cumulative frequencies found no change in head diameter among our hormone replacement con- 

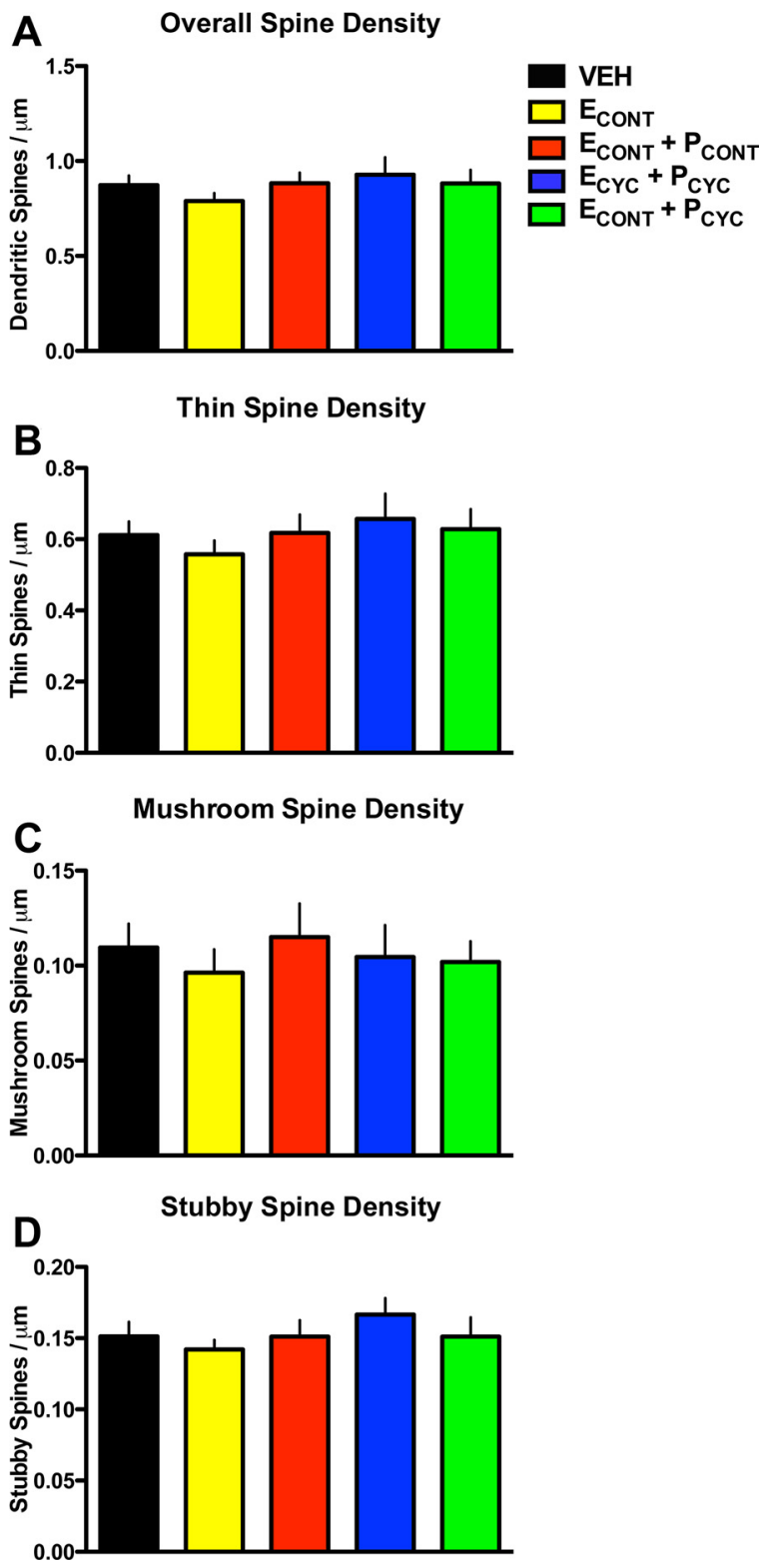

Figure 3. HT and dendritic spine density. $A$, Overall dendritic spine density on aged monkey dIPFC neurons. ANOVA found no significant differences in overall spine density between groups. $\boldsymbol{B}-\boldsymbol{D}$, Spine densities divided by spine types: thin $(\boldsymbol{B})$, mushroom $(\boldsymbol{C})$, and stubby $(\boldsymbol{D})$. ANOVA found no main effect on condition for any spine type density. See Results for details. ditions (Kolmogorov-Smirnov tests; $p>0.7$ for all comparisons; Fig. $4 A$ ). Analysis of spine volume also found no effect of hormonal condition on mean spine volume $\left(F_{(4,30)}=0.3258, p>\right.$ 0.8 ), and analysis of spine volume cumulative frequencies found no effect of HT (Kolmogorov-Smirnov tests; $p>0.8$ for all comparisons; Fig. $4 B)$.

\section{Discussion}

The present study was designed to determine the effects of several clinically relevant hormone replacement therapy regimens on dlPFC neurons in an aging NHP model. In aged, OVX female monkeys, we assessed the effects of HT on neuronal morphology using intracellular dye injections and quantitative laser microscopy of dlPFC pyramidal neurons in layer III. Despite the effective delivery of hormones as confirmed by serum hormone levels, the HTs used here failed to alter dlPFC global dendritic morphology, the density of dendritic spines, or spine morphology. These findings, in combination with detailed behavioral analyses of the same monkeys, which found no HT-related cognitive enhancement in delayed response (DR) performance (M. Baxter, M. Roberts, N. Gee, B. Lasley, J. Morrison, and P. Rapp, unpublished observations), have direct implications for the clinical use of HT in women.

The dynamic functions mediated by dlPFC have been suggested to be highly dependent on inputs at dendritic spines (Elston, 2000; Arnsten et al., 2010), and we hypothesize that a high level of synaptic structural plasticity and spine turnover are crucial components of the synaptic strategy used by dlPFC neuronal circuits. Previous studies using the same aged OVX NHP model have shown that cyclic $E$ injections enhanced performance on the dlPFC-dependent DR task alongside a concomitant increase of dendritic spine density on dlPFC neurons (Rapp et al., 2003; Hao et al., 2006, 2007). The E-induced spine density increase was primarily attributed to the density of thin dendritic spines, which we suspect are the critical spines for supporting optimal dIPFC function and the most vulnerable spine class to aging on dlPFC neurons (Dumitriu et al., 2010). These results demonstrate that dlPFC layer III pyramidal neurons remain viable therapeutic targets during aging and retain the capacity for spinogenesis in response to HT.

The current experiment was designed to test whether or not the timing (i.e., cyclic or continuous) and formulations (i.e., unopposed E or E with P) are critical determinants of HT's neurobiological benefits. The most prevalent form of HT prescribed to women, continuous $\mathrm{E}$ and $\mathrm{P}\left(\mathrm{E}_{\mathrm{CONT}}+\mathrm{P}_{\mathrm{CONT}}\right)$, failed to increase dendritic spine density or alter existing spine morphology in aged OVX NHPs; similar results were found with the other treatments tested (i.e.,
Figure 4. HT and dendritic spine morphology. $A$, Cumulative frequency plots of individual spine head diameters. KolmogorovSmirnov tests found no differences between groups ( $p>0.7$ for all pairwise comparisons). Similarly, mean spine head diamete was also unaffected by treatment (data not shown). $\boldsymbol{B}$, Cumulative frequency plots of individual spine head volume. KolmogorovSmirnov tests found no differences between groups ( $p>0.8$ for all pairwise comparisons). Similarly, mean spine volume did no differ between groups (data not shown). See Results for details. $\mathrm{E}_{\mathrm{CONT}}, \mathrm{E}_{\mathrm{CYC}}+\mathrm{P}_{\mathrm{CYC}}$, and $\mathrm{E}_{\mathrm{CONT}}+\mathrm{P}_{\mathrm{CYC}}$ ). We also found no change in the density or morphology of the thin spines, which are known to be highly motile (Holtmaat et al., 2005) and can be incorporated into neuronal circuitry with activity (Srivastava et al., 2008). Taken within the context of our previous findings (Hao et al., 2007), these findings strongly indicate that a cyclic schedule of $\mathrm{E}$ replacement, particularly when $\mathrm{E}$ is given every $21 \mathrm{~d}$, is more effective at inducing spinogenesis in aging PFC circuits compared with a continuous replacement. Behavioral data from aging

rodent studies have reported findings that

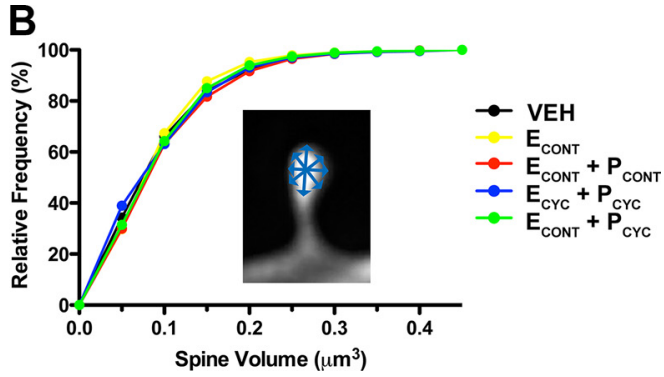


support this hypothesis; for example, continuous E was shown to be significantly less effective at enhancing WM performance than continuous E combined with cyclic E injections in aging female rats (Markowska and Savonenko, 2002). Such data imply that a decisive factor in both the behavioral and neurobiological effects of E replacement may be the cyclic engagement of the molecular machinery that mediate the effects of $\mathrm{E}$, including both genomic and nongenomic estrogen receptors, downstream signaling cascades, and NMDA-type glutamate receptors (for review, see Spencer et al., 2008).

The second substantial finding from the present study is that $\mathrm{P}$ has the ability to modulate the effectiveness of ET at inducing spine formation in aging dlPFC neurons. Previous studies have demonstrated that $\mathrm{P}$ can modulate several of the neurobiological effects of $\mathrm{E}$ treatment (for review, see Brinton et al., 2008), and our data extend this interaction to aging monkey dlPFC circuitry. Here, cyclic P replacement designed to simulate intact hormonal rhythms blocked the cyclic E-induced increase in spine density on aging dlPFC neurons. The implication that $\mathrm{P}$ might antagonize some of the beneficial effects of $\mathrm{E}$ are in agreement with data reported from rodent models, where $\mathrm{P}$ has been shown to diminish the effects of E on hippocampal spine formation (Woolley and McEwen, 1992, 1993; Murphy and Segal, 2000), neuroprotection (Jayaraman and Pike, 2009), gene transcription (BimonteNelson et al., 2004), and memory (Bimonte-Nelson et al., 2006). At the time of perfusion in the current study, levels of $P$ were essentially identical to OVX monkeys (data not shown); therefore, we interpret these findings to mean that P's modulatory effects on E-induced spinogenesis may be dependent on downstream actions of $\mathrm{P}$ treatment that persist after serum levels decline. Future studies focusing on NMDA receptors, which have been reported to be required for E-induced spinogenesis (Woolley and McEwen, 1994; McEwen et al., 1999), should provide fundamental data regarding potential mechanisms by which $\mathrm{P}$ might alter the effectiveness of $\mathrm{E}$ treatments.

In conclusion, the present study provides the first data regarding the importance of HT schedule and formulation on spine synapse structure in aging NHP neurons. Our results indicate that continuous $\mathrm{E}$ is ineffective at inducing spinogenesis in aging dlPFC, and that spinogenesis associated with cyclic ET may be blocked by concomitant $P$ replacement. Consequently, it is essential that future experiments aim to find the HT that optimally supports both the structure and function of aging dlPFC circuits, as well as other age-sensitive circuits in brain regions such as the hippocampus. Furthermore, continuing these investigations in the aging NHP model will expand translational efforts by reconciling the disparate effects HT produces across the human and preclinical paradigms.

\section{References}

Arnsten AF, Paspalas CD, Gamo NJ, Yang Y, Wang M (2010) Dynamic network connectivity: A new form of neuroplasticity. Trends Cogn Sci 14:365-375.

Bailey ME, Wang AC, Hao J, Janssen WG, Hara Y, Dumitriu D, Hof PR, Morrison JH (2011) Interactive effects of age and estrogen on cortical neurons: implications for cognitive aging. Neuroscience 191:148-158.

Bimonte-Nelson HA, Nelson ME, Granholm AC (2004) Progesterone counteracts estrogen-induced increases in neurotrophins in the aged female rat brain. Neuroreport 15:2659-2663.

Bimonte-Nelson HA, Francis KR, Umphlet CD, Granholm AC (2006) Progesterone reverses the spatial memory enhancements initiated by tonic and cyclic oestrogen therapy in middle-aged ovariectomized female rats. Eur J Neurosci 24:229-242.

Brinton RD, Thompson RF, Foy MR, Baudry M, Wang J, Finch CE, Morgan TE, Pike CJ, Mack WJ, Stanczyk FZ, Nilsen J (2008) Progesterone receptors: form and function in brain. Front Neuroendocrinol 29:313-339.

Dumitriu D, Hao J, Hara Y, Kaufmann J, Janssen WG, Lou W, Rapp PR, Morrison JH (2010) Selective changes in thin spine density and morphology in monkey prefrontal cortex correlate with aging-related cognitive impairment. J Neurosci 30:7507-7515.

Dumitriu D, Rodriguez A, Morrison JH (2011) High-throughput, detailed, cell-specific neuroanatomy of dendritic spines using microinjection and confocal microscopy. Nat Protoc 6:1391-1411.

Elston GN (2000) Pyramidal cells of the frontal lobe: all the more spinous to think with. J Neurosci 20:RC95.

Gibbs RB, Gabor R (2003) Estrogen and cognition: applying preclinical findings to clinical perspectives. J Neurosci Res 74:637-643.

Goldman-Rakic PS (1995) Cellular basis of working memory. Neuron 14:477-485.

Hao J, Rapp PR, Leffler AE, Leffler SR, Janssen WG, Lou W, McKay H, Roberts JA, Wearne SL, Hof PR, Morrison JH (2006) Estrogen alters spine number and morphology in prefrontal cortex of aged female rhesus monkeys. J Neurosci 26:2571-2578.

Hao J, Rapp PR, Janssen WG, Lou W, Lasley BL, Hof PR, Morrison JH (2007) Interactive effects of age and estrogen on cognition and pyramidal neurons in monkey prefrontal cortex. Proc Natl Acad Sci USA 104:11465-11470.

Holtmaat AJ, Trachtenberg JT, Wilbrecht L, Shepherd GM, Zhang X, Knott GW, Svoboda K (2005) Transient and persistent dendritic spines in the neocortex in vivo. Neuron 45:279-291.

Jayaraman A, Pike CJ (2009) Progesterone attenuates oestrogen neuroprotection via downregulation of oestrogen receptor expression in cultured neurones. J Neuroendocrinol 21:77-81.

Kimura D (1995) Estrogen replacement therapy may protect against intellectual decline in postmenopausal women. Horm Behav 29:312-321.

Lacreuse A (2006) Effects of ovarian hormones on cognitive function in nonhuman primates. Neuroscience 138:859-867.

Markowska AL, Savonenko AV (2002) Effectiveness of estrogen replacement in restoration of cognitive function after long-term estrogen withdrawal in aging rats. J Neurosci 22:10985-10995.

McEwen BS, Tanapat P, Weiland NG (1999) Inhibition of dendritic spine induction on hippocampal CA1 pyramidal neurons by a nonsteroidal estrogen antagonist in female rats. Endocrinology 140:1044-1047.

Moore TL, Killiany RJ, Herndon JG, Rosene DL, Moss MB (2006) Executive system dysfunction occurs as early as middle-age in the rhesus monkey. Neurobiol Aging 27:1484-1493.

Morrison JH, Brinton RD, Schmidt PJ, Gore AC (2006) Estrogen, menopause, and the aging brain: how basic neuroscience can inform hormone therapy in women. J Neurosci 26:10332-10348.

Murphy DD, Segal M (2000) Progesterone prevents estradiol-induced dendritic spine formation in cultured hippocampal neurons. Neuroendocrinology 72:133-143.

Phillips SM, Sherwin BB (1992) Effects of estrogen on memory function in surgically menopausal women. Psychoneuroendocrinology 17:485-495.

Rapp PR, Morrison JH, Roberts JA (2003) Cyclic estrogen replacement improves cognitive function in aged ovariectomized rhesus monkeys. J Neurosci 23:5708-5714.

Roberts JA, Gilardi KV, Lasley B, Rapp PR (1997) Reproductive senescence predicts cognitive decline in aged female monkeys. Neuroreport 8:2047-2051.

Shumaker SA, Legault C, Rapp SR, Thal L, Wallace RB, Ockene JK, Hendrix SL, Jones BN 3rd, Assaf AR, Jackson RD, Kotchen JM, WassertheilSmoller S, Wactawski-Wende J (2003) Estrogen plus progestin and the incidence of dementia and mild cognitive impairment in postmenopausal women: the Women's Health Initiative Memory Study: a randomized controlled trial. JAMA 289:2651-2662.

Spencer JL, Waters EM, Romeo RD, Wood GE, Milner TA, McEwen BS (2008) Uncovering the mechanisms of estrogen effects on hippocampal function. Front Neuroendocrinol 29:219-237.

Srivastava DP, Woolfrey K, Jones KA, Shum CY, Lash LL, Swanson GT, 
Penzes P (2008) Rapid enhancement of two-step wiring plasticity by estrogen and NMDA receptor activity. Proc Natl Acad Sci U S A 105:14650-14655.

Turgeon JL, McDonnell DP, Martin KA, Wise PM (2004) Hormone therapy: physiological complexity belies therapeutic simplicity. Science 304:1269-1273.

Verghese J, Kuslansky G, Katz MJ, Sliwinski M, Crystal HA, Buschke H, Lipton RB (2000) Cognitive performance in surgically menopausal women on estrogen. Neurology 55:872-874.

Voytko ML, Tinkler GP, Browne C, Tobin JR (2009) Neuroprotective effects of estrogen therapy for cognitive and neurobiological profiles of monkey models of menopause. Am J Primatol 71:794-801.
Wang M, Gamo NJ, Yang Y, Jin LE, Wang XJ, Laubach M, Mazer JA, Lee D, Arnsten AF (2011) Neuronal basis of age-related working memory decline. Nature 476:210-213.

Woolley CS, McEwen BS (1992) Estradiol mediates fluctuation in hippocampal synapse density during the estrous cycle in the adult rat. J Neurosci 12:2549-2554.

Woolley CS, McEwen BS (1993) Roles of estradiol and progesterone in regulation of hippocampal dendritic spine density during the estrous cycle in the rat. J Comp Neurol 336:293-306.

Woolley CS, McEwen BS (1994) Estradiol regulates hippocampal dendritic spine density via an $\mathrm{N}$-methyl-D-aspartate receptor-dependent mechanism. J Neurosci 14:7680-7687. 